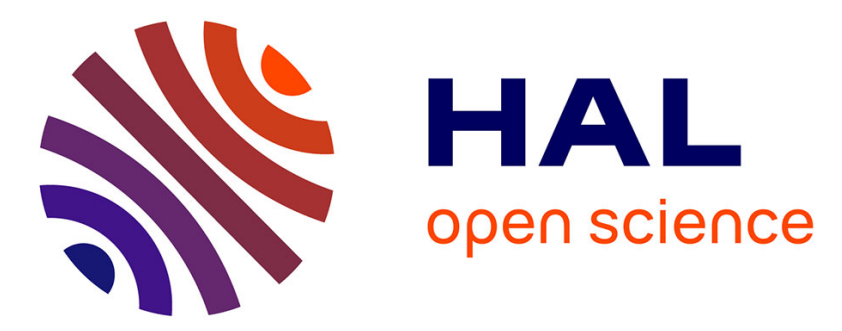

\title{
Preventable deaths in a French regional trauma system: A six-year analysis of severe trauma mortality
}

\author{
Edouard Girard, Q. Jegousso, B. Boussat, P. Francois, F.-X. Ageron, C.
}

Letoublon, P. Bouzat

\section{To cite this version:}

Edouard Girard, Q. Jegousso, B. Boussat, P. Francois, F.-X. Ageron, et al.. Preventable deaths in a French regional trauma system: A six-year analysis of severe trauma mortality. Journal of Visceral Surgery, 2019, 156 (1), pp.10-16. 10.1016/j.jviscsurg.2018.05.002 . hal-02064522

\section{HAL Id: hal-02064522 \\ https://hal.science/hal-02064522}

Submitted on 22 Oct 2021

HAL is a multi-disciplinary open access archive for the deposit and dissemination of scientific research documents, whether they are published or not. The documents may come from teaching and research institutions in France or abroad, or from public or private research centers.
L'archive ouverte pluridisciplinaire HAL, est destinée au dépôt et à la diffusion de documents scientifiques de niveau recherche, publiés ou non, émanant des établissements d'enseignement et de recherche français ou étrangers, des laboratoires publics ou privés.

\section{(ㄷ)(1) $\$$}

Distributed under a Creative Commons Attribution - NonCommercial| 4.0 International 
Preventable deaths in a French regional trauma system: a six-year analysis of severe trauma mortality.

Edouard Girard, MD ${ }^{1,2}$

Quentin Jegousso, $\mathrm{MD}^{3}$

Bastien Boussat, MD 2,4 $^{2,4}$

Patrice François, MD, $\mathrm{PhD}^{2,4}$

François-Xavier Ageron, $\mathrm{MD}^{5}$

Christian Letoublon, $\mathrm{MD}, \mathrm{PhD}^{1,2}$

Pierre Bouzat MD, $\mathrm{PhD}{ }^{2,3}$

For the TRENAU group*

1. Department of emergency surgery and visceral surgery, Grenoble University Hospital, F38000 Grenoble, France

2. Grenoble Alps University, F-38000 Grenoble, France

3. Grenoble Alps Trauma centre, Department of anaesthesiology and intensive care medicine, Grenoble University Hospital, F-38000, Grenoble, France

4. Quality of care unit, Grenoble University Hospital, F-38000 Grenoble, Grenoble, France

5. Department of emergency medicine, Annecy Hospital, F-74000 Annecy, France

* Collaborators (TRENAU Group): see supplemental file $\mathbf{n}^{\circ} \mathbf{1}$

Correspondence to Dr Edouard GIRARD, Département de chirurgie digestive et de l'urgence, Hôpital Albert Michallon, BP 217, F-38043 Grenoble, France.

Tel : + 33(0)4 76765428 Fax : 04767658 02. E-mail: Egirard1@chu-grenoble.fr 


\section{Abstract}

Background: Analyzing mortality in a mature trauma system is useful to improve quality of care of severe trauma patients. Standardization of error reporting can be done using the classification of the Joint Commission on the Accreditation of Healthcare Organizations (JCAHO). The aim of our study was to describe preventable deaths in our trauma system and to classify errors according to the JCAHO taxonomy.

Methods: We performed a six-year retrospective study using the registry of the Northern French Alps trauma network (TRENAU). Consecutive patients who died in the prehospital field or within their stay at hospital were included. An adjudication committee analyzed deaths to identify preventable or potentially preventable deaths from 2009 to 2014. All errors were classified using the JCAHO taxonomy.

Results: Within the study period, 503 deaths were reported among 7,484 consecutive severe trauma patients (overall mortality equal to $6.7 \%$ ). Seventy-two (14\%) deaths were judged as potentially preventable and $36(7 \%)$ deaths as preventable. Using the JACHO taxonomy, 170 errors were reported. These errors were detected both in the prehospital setting and in the hospital phase. Most were related to clinical performance of physicians and consisted of rulebased or knowledge based failures. Prevention or mitigation of errors required an improvement of communication among caregivers.

Conclusions: Standardization of error reporting is the first step to improve the efficiency of trauma systems. Preventable deaths are frequently related to clinical performance in the early phase of trauma management. Universal strategies are necessary to prevent or mitigate these errors.

Keywords: trauma system; preventable deaths; avoidable errors; quality; safety 


\section{Background}

Over the past decades, the management of severe trauma patients has been improved by the implementation of trauma systems across the world allowing standardization of trauma care [1]. Trauma systems are based upon early recognition of severe trauma, adequate allocation of resources for each patient and the standardization of procedures for trauma care. Indicators for a trauma system's effectiveness rely on the rate of under/over-triage [2] but also on the analysis of mortality within the trauma network [3]. Classically, deaths after severe trauma can be categorized as followed: preventable deaths, potentially preventable deaths and non-preventable deaths [4-6]. This categorization can be performed by benchmarking care to accepted guidelines (For instance, advanced trauma life support [7] or National Institute for health and Care Excellence, guidelines for major trauma) or by determining the risk of death according to trauma scores (Trauma Revised Injury Severity Score, TRISS or mechanism, age, Glasgow coma scale, arterial pressure, MGAP, score) [8, 9]. Regardless of methodology, the concept of preventable deaths has been reported for decades in severe trauma patients and reducing their number is one of the main objectives of trauma system [10]. Hence, the analysis of errors related to preventable/potentially preventable deaths is necessary for quality improvement of trauma care within a specific region. Ultimately, comparisons of errors between trauma systems would be useful to analyze their efficiency and thereby defining the best standard of care. For that purpose, analyzing preventable and potentially preventable deaths is of interest to classify reported errors that may induce corrective actions to avoid these events. A major limitation for work in this area is the lack of standardization of error classification, which undermines the reproducibility of such analysis. To overcome this limit, the Joint Commission on the accreditation of Healthcare Organizations (JCAHO) has proposed a taxonomy to facilitate a common approach for patient safety information systems [11]. This classification allocates errors in five interacting nodes: impact, type, domain, cause 
and prevention. The JCAHO taxonomy has been successfully used for the analysis of preventable deaths in diverse trauma systems and has become the benchmark of error reporting after severe trauma $[6,12,13]$. Since 2007, a French regional trauma system, named TRENAU, has been implemented in the French Alps to optimize the management of trauma patients in this region [14]. Our organization has demonstrated efficiency in terms of under and over-triage but an analysis of mortality has not been performed [15]. The main objective of our study was to describe preventable/potentially preventable deaths in our trauma system and to classify our errors using the JCAHO taxonomy. 


\section{Methods}

\section{Study design and data collection}

We conducted a retrospective study using the registry of the Trauma System of the Northern French Alps (TRENAU). Briefly, this trauma system gathers data from thirteen hospitals located in the Northern French Alps and consists of one level-I trauma center, two level-II trauma centers and ten level-III trauma centers [14]. A registry has been implemented since 2009 and has been collected data prospectively using the Utstein-style template [16]. The Regional Institutional Ethics Committee approved the implementation of the TRENAU registry (Comité d'Ethique des Centres d'Investigation Clinique de l'inter-région Rhône-Alpes-Auvergne, IRB number 5891) and, given its observational nature, waived the requirements for written informed consent from each patient.

Consecutive adult patients recorded in the TRENAU registry from January 2009 to December 2014 were included if they died following a severe traumatic injury in the prehospital setting or within their stay at hospital. Patients who died before the arrival of prehospital emergency physicians were not included in the TRENAU registry and, thus, were not considered for this study. The following data were collected for each patient: age, sex, mechanism, first recorded vital signs, the mechanism, Glasgow coma scale (GCS), age and arterial pressure (MGAP) score [9] in the pre-hospital setting, vital signs on admission, the need for emergency surgery or embolization, the injury severity score (ISS) and the Trauma revised injury severity score (TRISS) [8]. The Injury Severity Score (ISS) is an anatomical scoring system that provides an overall score for patients with multiple injuries. Each injury is assigned an Abbreviated Injury Scale (AIS) score and is allocated to one of six body regions (Head, Face, Chest, Abdomen, Extremities (including Pelvis), External). Only the highest AIS 
score in each body region is used. The three most severely injured body regions have their score squared and added together to produce the ISS score. The ISS score correlates linearly with mortality, morbidity, hospital stay and other measures of severity. The TRISS determines the probability of survival. It is calculated from the ISS, the Revised Trauma Score (calculated with respiratory rate, GCS and systolic arterial blood pressure) and patient's age. Cause, date and location (prehospital phase, trauma bay or intensive care unit, ICU) of death were also reported.

\section{Primary endpoint}

The primary endpoint was the preventability of death from the pre-hospital field to the end of hospital's stay. Each patient's file was reviewed by a multidisciplinary independent committee (adjudication committee) composed by two anesthesiologists (PB, QJ) and two trauma surgeons (EG, CL) to classify mortality according to the analysis of errors. A preventable death was defined by a death caused directly by an avoidable error. A potentially preventable death was defined by a death that might have been caused by a preventable error, and non-preventable deaths were those that occurred regardless of any errors in the patient's management. Errors were also classified according to the Joint Commission on the accreditation of Healthcare Organizations (JCAHO) taxonomy [11].

\section{Secondary endpoints}

Secondary endpoints were 1) the comparison between patients with preventable/potentially preventable deaths and patients with non-preventable deaths and 2) the time course of preventable/potentially preventable deaths from 2009 to 2014. 
Statistical analysis

Continuous variables were presented as median and 25th-75th interquartile ranges (IQRs). Categorical variables were presented as numbers and percentages. We compared patient characteristics according to the preventability of death using a Mann-Whitney test for continuous variables and a Chi square test, or a Fisher exact test where appropriate, for categorical variables. The trend in mortality over time was tested with a Chi-squared test for linear trend. Subgroup trends according to deaths' category were also analyzed. Two-sided p values $<0.05$ were considered as statistically significant. All analyses were performed using Stata version 14.0 (Stata Corporation, College Station, TX, USA). 


\section{Results}

Within the study period, 7,484 consecutive trauma patients were included in the TRENAU registry. Among them, 503 consecutive patients died (overall mortality equal to $6.7 \%)$ and were included in the study. Twenty-nine patients $(6 \%)$ died in the prehospital phase, $47(9 \%)$ patients died in the trauma bay before their transfer to ICU, and $427(85 \%)$ patients died in the ICU. One hundred and sixty-eight patients (33\%) died within the first 24 hours after the insult and median time between accident and death was 1 [0-5] day. Seventytwo (14\%) deaths were judged as potentially preventable and $36(7 \%)$ deaths as preventable. Patients characteristics are shown in Table $\mathbf{1}$ and causes of deaths are presented in Supplemental File $\mathbf{N}^{\circ}$ 2. Patients with preventable/potentially preventable deaths had different location of lethal injuries as compared to patients with non-preventable deaths. Nonpreventable deaths were most often attributed to CNS injuries, whereas preventable deaths were related to torso or pelvic injuries with acute hemorrhage. Accordingly, pre-hospital GCS were also lower in patients with non-preventable deaths. At hospital admission, GCS were similar between the two groups due to shock or on-going sedation. MGAP score and TRISS scores were also most severe in these patients. Regarding the time course of preventable/potentially preventable deaths, the proportion of patients with preventable deaths remained stable from 2009 to 2014 . Indeed, the rate of preventable/potentially preventable deaths was 28\% in 2009 and 25\% in 2014 ( $p=0.43$; see Supplemental File $\mathbf{N}^{\circ}$ 2).

We reported 170 errors in 108 patients (92 errors in preventable deaths and 78 errors in potentially preventable deaths, Table 2). Errors were both described in the pre-hospital setting and in the in-hospital phases. Excessive pre-hospital time was the prominent error found for preventable deaths whereas triage errors and omission of essential procedures were 
the most frequent types of error for potentially preventable deaths. Using the classification of errors in the JCAHO taxonomy, errors in clinical performance were prominent. They mainly occurred during the diagnostic process or within the therapeutic procedure (Table 3) and consisted of incorrect procedures or delayed correct procedures. Regarding the setting of errors, most of them were found in the pre-hospital phase (Table 4). Main errors consisted of inadequate triage (failure in diagnosis) or excessive pre-hospital time. Errors could also occur in the trauma bay or in the operating room and were related to inappropriate diagnostic procedures. For instance, we reported 24 errors in the operating room for 23 patients. Three types of errors were found: excessive delay for surgery, technical difficulties, wrong procedures or failure to perform damage control procedures. Regarding timing of surgery, these errors concerned a delayed evacuation of a subdural hematoma, or delayed laparotomies. Regarding technical difficulties, we found an injury of the mesenteric artery and an iliac venous wound. Regarding therapeutic mistakes, some laparotomies were unjustified because of negative FAST. In other cases, some procedures were carried out not respecting damage control rules: operating time too long, complete abdominal parietal closure, no pelvic packing, no peri-hepatic packing, or no vascular damage control on lower limbs. Wrong procedures in this context were incorrect surgical incisions like sternotomy for a thoracic damage control surgery. The main cause of error was human failure, specifically knowledgebased and rule-based errors. These mistakes were related to an inadequate observance or an ignorance to established instructions or protocols (Table 5), for instance, performing CT scan in hemodynamically unstable patients.

Prevention or mitigation of errors required an improvement of communication among caregivers (Supplemental File $\mathbf{N}^{\circ} \mathbf{3}$ ). This prevention measure lies upon regular meetings between anesthesiologists, emergency physicians, radiologists and trauma surgeons to discuss morbidity and mortality. Prevention or mitigation of errors is also based upon the diffusion of 
procedures within the trauma network. Selective measures, like eliminating wrong procedures, were less frequently necessary to avoid errors in prehospital triage or to establish the appropriate surgical technique in the operating room. 


\section{Discussion}

The analysis of mortality is mandatory to evaluate the efficiency of a trauma system and the rate of preventable or potentially preventable deaths is increasingly used as a benchmark of patient safety reporting in trauma systems across the world. Over a 6-year period including 7,484 trauma patients, we found 170 errors in 108 preventable/potentially preventable deaths among 503 deaths in our regional trauma system. Using a common classification, the prominent type of error was a failure in clinical performance of physicians in the prehospital setting or in the trauma bay. These mistakes were mainly made during diagnostic or therapeutic procedures and the majority of errors were related to rule-based or knowledge-based failures.

Improving the quality of care in mature trauma systems is the ultimate goal of these organizations. The first step to perform such improvement is undoubtedly linked to the recognition of errors, which prompts corrective actions to limit future potential failures [6]. Despite this well-known concept, few studies addressed appropriately the challenge of errors' classification. In the United States, the Pennsylvania trauma system and the Virginia Commonwealth University Medical Centre used the JACHO classification for errors reporting[6, 13], while others used their own classification $[10,17]$. The disparity of error reporting does not facilitate comparisons between trauma systems whereas trauma procedures may be optimized by such analysis. In Europe, the JACHO taxonomy was also used in the United Kingdom and Spain to categorize errors during the management of severe trauma patients $[12,18]$. Our study adds to a small body of literature regarding classification of avoidable errors in preventable trauma deaths. Preventable or potentially preventable deaths account from $6 \%$ to $27 \%$ of deaths in trauma systems [5, 6, 13]. With $21 \%$ of 
preventable/potentially preventable deaths, our study displayed poor performance of our trauma system according to the existing literature. The variability of these preventable deaths relies upon the efficiency of trauma system but also upon the methodology used by the adjudication committee. Similarly, the vast majority of previously published reports reviewed patients' files with an expert panel to decide whether trauma death was categorized as preventable, potentially preventable or non-preventable $[10,13]$. This methodology did not account for uncertainty in the classification of potentially preventable death, and using a 100point scale, Kobewka et al showed that only 5 of 31 deaths classified as a "possibly preventable" were judged to likely be alive in 3 months with perfect care [19]. In our study, we had only $7 \%$ of preventable deaths whereas $14 \%$ of deaths were judged as potentially preventable by the expert committee. The high proportion of preventable/potentially preventable deaths in our study was probably related to the high proportion of potentially preventable deaths that was subjected to uncertainty. Alternatively, the proportion of preventable deaths was similar to other studies and overall mortality $(6.7 \%)$ in the TRENAU was also comparable.

In our study, we found 170 errors occurring in 108 preventable/potentially preventable trauma deaths. Interestingly, the setting of these errors was mainly in the prehospital field. On-scene treatment and diagnosis were performed by emergency physicians in our trauma system [14] and such findings challenged the performance of pre-hospital medical teams. The main error was excessive pre-hospital time, based on a one-hour time-lapse. It should be noted that the French prehospital organization is different from the US prehospital system. In France, emergency physicians are in charge of the prehospital management of severe trauma whereas paramedics lead this management in the US. Such difference may explain longer stay in the prehospital field where on-site damage control resuscitation can be provided. Another frequent mistake was an inadequate triage due to ignorance of existing guidelines in our 
network [15]. These findings highlight the need for continuous education of pre-hospital physicians but also do not reflect actual knowledge of prehospital doctors as intense education regarding damage control resuscitation has only been done since recent French terrorist attacks [20]. Moreover, the TRENAU network is located in a mountainous area where conditions for pre-hospital teams may be hazardous [14]. These geographical considerations may account for inherent excessive pre-hospital time due to extreme conditions or complex extraction. In the trauma bay or in the operating room, we also found errors mainly related to omissions of essential procedure. For instance, unstable patients have been transferred for CT scanning or damage control protocols have not been applied. These errors were surprising since intra-hospital management might be seen as a more controlled scenario. They occurred within the trauma bay or within the operating room and most common errors were clinical and mainly therapeutic. These failures were observed in trauma leaders who did not comply or ignored established protocols. Although meetings were frequently organized between emergency physicians, anesthesiologists, trauma surgeons and radiologists from 2009 to 2014, these findings proved that the diffusion of procedures remained challenging in trauma centers. Accordingly, prevention of errors was mainly based upon improving communication between caregivers to ensure adequate compliance to procedures and protocols. These universal measures were more frequent than selective measures directed to different subgroup of trauma patients. Of note, the human factor was highly involved in these mistakes. This finding highlighted the critical importance of effective teamwork and communication in delivering a high-quality patient care. Our findings also highlight the importance of initial and continuous training in trauma care for anesthesiologists, surgeons, and emergency physicians. Several university diplomas for severe trauma management exist in France and the compliance to procedures may be enhanced by such initiatives. 
We acknowledge several limitations of our study. First, the number of deaths was relatively small and only 170 errors were described. Nevertheless, the study size was similar to previously published works and a 6-year analysis allowed us to cover a large period of time within our trauma system. Second, our methodology to categorize deaths was based upon an adjudication committee composed by two anesthesiologists and two trauma surgeons. This method does not account for uncertainty in categorization and may have contributed to excessive number of potentially preventable deaths. However, the JACHO taxonomy was applied and the classification of errors was reliable enough to allow comparisons between trauma systems. 


\section{Conclusions}

With our 6-year retrospective study, we demonstrated that preventable or potentially preventable deaths were related to avoidable errors in the prehospital field or in the trauma bay. These errors were attributed to physicians who ignored essential procedures regarding diagnosis or therapy. These results confirm that preventable deaths are an indicator of quality of care in an organized trauma system. Efforts should be made to reduce their prevalence and the first step is probably to improve communication among caregivers. Apart from selfevaluation, describing errors is also helpful for determining the best management of severe trauma patients in developed countries. 


\section{Competing interest}

The authors declare that they have no competing interest.

\section{Acknowledgements}

The authors thank the investigators of the TRENAU network for implementing the registry.

\section{Declarations}

The Regional Institutional Ethics Committee approved the implementation of the TRENAU registry (Comité d'Ethique des Centres d'Investigation Clinique de l'inter-région Rhône-Alpes-Auvergne, IRB number 5891) and, given its observational nature, waived the requirements for written informed consent from each patient

Consent for publication: not applicable.

Availability of data and supporting materials section: the authors can share their data if requested by any reader of the present study.

Fundings: This research did not receive any specific grant from funding agencies in the public, commercial, or not-for-profit sectors 


\section{References}

[1] MacKenzie EJ, Rivara FP, Jurkovich GJ, Nathens AB, Frey KP, Egleston BL, et al. A national evaluation of the effect of trauma-center care on mortality. $\mathrm{N}$ Engl $\mathrm{J}$ Med. 2006;354:366-78.

[2] Hospital and prehospital resources for optimal care of the injured patient. Committee on Trauma of the American College of Surgeons. Bull Am Coll Surg. 1986;71:4-23.

[3] Shafi S, Friese R, Gentilello LM. Moving beyond personnel and process: a case for incorporating outcome measures in the trauma center designation process. Arch Surg. 2008;143:115-9; discussion 20.

[4] Davis JW, Hoyt DB, McArdle MS, Mackersie RC, Shackford SR, Eastman AB. The significance of critical care errors in causing preventable death in trauma patients in a trauma system. J Trauma. 1991;31:813-8; discussion 8-9.

[5] Teixeira PG, Inaba K, Hadjizacharia P, Brown C, Salim A, Rhee P, et al. Preventable or potentially preventable mortality at a mature trauma center. J Trauma. 2007;63:1338-46; discussion 46-7.

[6] Vioque SM, Kim PK, McMaster J, Gallagher J, Allen SR, Holena DN, et al. Classifying errors in preventable and potentially preventable trauma deaths: a 9-year review using the Joint Commission's standardized methodology. Am J Surg. 2014;208:187-94.

[7] Subcommittee A, American College of Surgeons' Committee on T, International Awg. Advanced trauma life support (ATLS(R)): the ninth edition. J Trauma Acute Care Surg. 2013;74:1363-6.

[8] Schluter PJ, Nathens A, Neal ML, Goble S, Cameron CM, Davey TM, et al. Trauma and Injury Severity Score (TRISS) coefficients 2009 revision. J Trauma. 2010;68:761-70. 
[9] Sartorius D, Le Manach Y, David JS, Rancurel E, Smail N, Thicoipe M, et al. Mechanism, glasgow coma scale, age, and arterial pressure (MGAP): a new simple prehospital triage score to predict mortality in trauma patients. Crit Care Med. 2010;38:831-7.

[10] Teixeira PG, Inaba K, Salim A, Rhee P, Brown C, Browder T, et al. Preventable morbidity at a mature trauma center. Arch Surg. 2009;144:536-41; discussion 41-2.

[11] Chang A, Schyve PM, Croteau RJ, O'Leary DS, Loeb JM. The JCAHO patient safety event taxonomy: a standardized terminology and classification schema for near misses and adverse events. Int J Qual Health Care. 2005;17:95-105.

[12] Montmany S, Pallisera A, Rebasa P, Campos A, Colilles C, Luna A, et al. Preventable deaths and potentially preventable deaths. What are our errors? Injury. 2016;47:669-73.

[13] Ivatury RR, Guilford K, Malhotra AK, Duane T, Aboutanos M, Martin N. Patient safety in trauma: maximal impact management errors at a level I trauma center. J Trauma. 2008;64:265-70; discussion 70-2.

[14] Bouzat P, Broux C, Ageron FX, Thony F, Arvieux C, Tonetti J, et al. Trauma network for severely injured patients. Ann Fr Anesth Reanim. 2013;32:531-4.

[15] Bouzat P, Ageron FX, Brun J, Levrat A, Berthet M, Rancurel E, et al. A regional trauma system to optimize the pre-hospital triage of trauma patients. Crit Care. 2015;19:111.

[16] Soreide E, Steen PA. New recommendations for uniform reporting of data following major trauma: "the Utstein style". Acta Anaesthesiol Scand. 2000;44:359-60.

[17] Esposito TJ, Sanddal ND, Hansen JD, Reynolds S. Analysis of preventable trauma deaths and inappropriate trauma care in a rural state. J Trauma. 1995;39:955-62.

[18] Pucher PH, Aggarwal R, Twaij A, Batrick N, Jenkins M, Darzi A. Identifying and addressing preventable process errors in trauma care. World J Surg. 2013;37:752-8. 
[19] Kobewka DM, van Walraven C, Taljaard M, Ronksley P, Forster AJ. The prevalence of potentially preventable deaths in an acute care hospital: A retrospective cohort. Medicine (Baltimore). 2017;96:e6162.

[20] Hirsch M, Carli P, Nizard R, Riou B, Baroudjian B, Baubet T, et al. The medical response to multisite terrorist attacks in Paris. Lancet. 2015;386:2535-8. 
Table 1. Population characteristics

\begin{tabular}{|c|c|c|c|}
\hline Variable & $\begin{array}{c}\text { Deaths } \\
(\mathrm{n}=503 \text { patients })\end{array}$ & $\begin{array}{l}\text { Non-preventable } \\
\text { deaths } \\
(n=395 \text { patients })\end{array}$ & $\begin{array}{c}\text { Preventable or } \\
\text { potentially } \\
\text { preventable deaths } \\
(\mathrm{n}=108 \text { patients })\end{array}$ \\
\hline Age, years & 55 [29-73] & $56[28-73]$ & $54[32-73]$ \\
\hline Male, n (\%) & $366(73)$ & $287(78)$ & $79(73)$ \\
\hline Penetrating trauma, n (\%) & $30(6)$ & $21(5)$ & $9(8)$ \\
\hline \multicolumn{4}{|l|}{ Pre-hospital vital signs } \\
\hline Glasgow coma score & $4[3-10]$ & $3[3-6]$ & $12[4-15]^{*}$ \\
\hline Systolic blood pressure, $\mathrm{mmHg}$ & $111[77-140]$ & 115 [80-140] & 99 [70-125] \\
\hline Heart rate, beats/min & 83 [60-110] & 80 [53-108] & $97[72-120]^{*}$ \\
\hline Pulse oximetry, $\%$ & 95 [86-99] & 94 [85-99] & $96[91-98]$ \\
\hline Pre-hospital MGAP score & $16[13-21]$ & $15[12-18]$ & $22[15-24]^{*}$ \\
\hline Low risk death, $\mathrm{n}(\%)$ & $60(13)$ & $25(7)$ & $35(37)$ \\
\hline Intermediate risk, $\mathrm{n}(\%)$ & $72(16)$ & $48(13)$ & $24(26)$ \\
\hline High risk death, $\mathrm{n}(\%)$ & $321(71)$ & $286(80)$ & $35(37)$ \\
\hline \multicolumn{4}{|l|}{ Vital signs on admission } \\
\hline Glasgow coma score & $3[3-4]$ & $3[3-3]$ & $3[3-15]$ \\
\hline Systolic blood pressure, $\mathrm{mmHg}$ & $110[85-130]$ & 115 [89-140] & 97 [70-110] \\
\hline Heart rate, beats/min & 83 [63-106] & 80 [63-104] & 90 [70-120] \\
\hline Pulse oximetry, $\%$ & 99 [94-100] & $100[95-100]$ & $97[89-100]$ \\
\hline Emergency surgery, n (\%) & $118(23)$ & $82(21)$ & $36(33)$ \\
\hline Embolization, n (\%) & $34(7)$ & $20(5)$ & $14(13)$ \\
\hline ISS & $29[25-43]$ & $29[25-41]$ & $30[20-50]$ \\
\hline
\end{tabular}

Main injured area 


$\begin{array}{rccc}\text { Central nervous system } & 363(72) & 339(86) & 24(22)^{*} \\ \text { Thorax } & 82(16) & 38(10) & 44(41)^{*} \\ \text { Abdomen } & 25(5) & 5(1) & 20(19)^{*} \\ \text { Pelvis } & 28(6) & 9(2) & 19(18)^{*} \\ \text { Limbs } & 5(1) & 4(1) & 1(1)\end{array}$

TRISS

$0.45[0.13 ; 0.79] \quad 0.42[0.13-0.69] \quad 0.72[0.19-0.91]^{*}$

Values are median [25th-75 ${ }^{\text {th }}$ interquartiles]. ISS: Injury severity score; MGAP: mechanism, age, Glasgow coma scale and arterial pressure; TRISS: trauma revised injury severity score. ${ }^{*}$ p-value < 0.05 vs non-preventable deaths. 
Table 2. Analysis of errors by the adjudication committee

\begin{tabular}{|c|c|c|c|}
\hline & $\begin{array}{l}\text { Preventable deaths } \\
\qquad n=72 \text { patients }\end{array}$ & $\begin{array}{c}\text { Potentially } \\
\text { preventable deaths } \\
\mathrm{n}=36 \text { patients }\end{array}$ & $\begin{array}{l}\text { Total } \\
\mathrm{n}=108 \text { patients }\end{array}$ \\
\hline Triage error & 8 & 14 & 22 \\
\hline Excessive pre-hospital time & 28 & 9 & 37 \\
\hline Incorrect pre-hospital treatment & 2 & 5 & 7 \\
\hline Inaccurate diagnosis & 9 & 11 & 20 \\
\hline Diagnosis delay & 5 & 7 & 12 \\
\hline Deaths during CT scanning & 2 & 7 & 9 \\
\hline Incorrect treatment at hospital & 10 & 10 & 20 \\
\hline Incorrect airway control & 6 & 1 & 7 \\
\hline Omission of essential procedure & 21 & 13 & 34 \\
\hline Accidental drain/catheter removal & 1 & 0 & 1 \\
\hline Equipment failure & 0 & 1 & 1 \\
\hline Total & 92 & 78 & 170 \\
\hline
\end{tabular}

Note: One preventable/potentially preventable death may be related to more than one error, so that sum totals of errors exceed the number of deaths. 
Table 3. Types of error according to JCAHO taxonomy

Types of errors Number

\section{Communication}

Inaccurate \& incomplete information $\quad 19$

$\begin{array}{ll}\text { Questionable advice or interpretation } & 7\end{array}$

Questionable documentation 5

Patient Management

Questionable tracking or follow-up $\quad 16$

Questionable referral or consultation 20

Questionable use of resources $\quad 64$

Clinical performance

Diagnostic (pre-intervention)

Correct diagnosis, questionable intervention 2

Inaccurate diagnosis 4

Incomplete diagnosis 29

Intervention

Correct procedure, with complication 2

Correct procedure, incorrectly performed 5

Correct procedure, but untimely 36

Omission if essential procedure $\quad 17$

Procedure contraindicated 9

Procedure not indicated 2

Post-intervention

Questionable prognosis 5

Note: Errors may involve multiple categories, sum totals do not equal 170. 
Table 4. Domain of error according to JCAHO taxonomy

\begin{tabular}{lc} 
Domain of errors & Number \\
\hline Setting & 10 \\
Emergency department & 40 \\
Diagnostic procedures (Crash room) & 24 \\
Operating room & 5 \\
Interventional radiology & 82 \\
Pre-Hospital care & 8 \\
Intensive care unit & \\
Staff & 165 \\
Physician & 5 \\
Nurse/paramedics & \\
Target & 105 \\
Therapeutic & 41 \\
Diagnostic & 24 \\
Other &
\end{tabular}

Note: Domain parameters can overlap so that errors can be classified in more than one category 
Table 5. Cause of error according to JCAHO taxonomy

Cause of error

Total

Organizational system

External*

6

Management

Organizational culture of safety

8

Protocols/Processes/Procedures

9

Oversight

Technical System

Equipment/Material Obsolescence/Availability

External*

Human

Patient factors $\dagger$

External*

Practitioner

Skill-based (failure in execution of stored instructions)

Rule-based (failure of recall of stored instructions) 80

Knowledge-based (insufficient time, incomplete knowledge) 28

Unclassifiable

Others causes

Negligence $\ddagger$

Recklessness§

*Failures that are beyond the control and responsibility of the organization

$\dagger$ Failures related to patient characteristics or actions that are beyond the control of the practitioner $\ddagger$ Failure to perform at the level of competence consistent with professional norms of practice and operation

$\S$ Intentional deviation from professional norms of good practice and operation without cause

Note: Cause parameters can overlap so that errors can be categorized in more than one category 